\title{
Biomechanics of Esophageal Function in Eosinophilic Esophagitis
}

\author{
Andrew J Read* and John E Pandolfino \\ Department of Medicine, Feinberg School of Medicine, Northwestern University, Chicago, Illinois, USA
}

Eosinophilic Esophagitis (EOE) is a chronic inflammatory disease of the esophagus triggered by an immune response that leads to symptoms of dysphagia, chest pain, and food impaction. EoE is a clinicopathologic syndrome that requires clinical symptoms and pathologic findings for a diagnosis. The inflammatory process and eosinophilic infiltration of the esophagus in EoE lead to fibrosis and structural changes within the esophagus that cause esophageal dysfunction. The biomechanics of the esophageal function in EoE have been explored using manometry, impedance planimetry, barium esophagograms, and endoscopic ultrasound. These studies have identified several biomechanical changes to the esophagus in EoE including pan-esophageal pressurization on manometry, changes in esophageal compliance with decreased distentisbility by impedance planimetry, decreased esophageal luminal diameter by esophagograms, and dysfunction in the esophageal longitudinal muscles by endoscopic ultrasound. Treatments for the disease involve dietary changes, immunosuppressive drugs, and dilation techniques. However, the data regarding the effect of these therapies on altering mechanical properties of the esophagus is limited. As the pathogenesis of esophageal dysfunction in EoE appears multifactorial, further study of the biomechanics of EoE is critical to better diagnose, monitor and treat the disease.

(J Neurogastroenterol Motil 2012;18:357-364)

\section{Key Words}

Biomechanics; Dilatation; Eosinophilic esophagitis; Manometry; Physiopathology

\section{Introduction}

Eosinophilic esophagitis (EoE) is an emerging disease characterized pathologically by eosinophilic infiltration of the esophagus and clinically by esophageal dysfunction that manifests as dysphagia, chest pain, and food impaction. ${ }^{1} \mathrm{EoE}$ is a chronic disease triggered by an immune response to food or environmental antigens in susceptible individuals. In EoE, the esophagus is infiltrated by eosinophils and a variety of structural and functional changes occur that lead to symptoms of esophageal dysfunction. While EoE may present with changes in the endoscopic appearance of the esophagus, the gross endoscopic appearance may be normal and the diagnosis may be uncovered only by biopsy. ${ }^{2}$ Despite normal findings on endoscopy, these patients may still harbor dysfunction in the esophageal body that would impair body wall mechanics.

The study of EoE is inherently a multidisciplinary field as it affects both pediatric and adult populations, bridging gastroenterology, allergy/immunology and pathology. Recent consen-

Received: June 12, 2012 Revised: None Accepted: July 1, 2012

(c) This is an Open Access article distributed under the terms of the Creative Commons Attribution Non-Commercial License (http://creativecommons. org/licenses/by-nc/3.0) which permits unrestricted non-commercial use, distribution, and reproduction in any medium, provided the original work is properly cited.

*Correspondence: Andrew J Read, MD

Division of Gastroenterology, Department of Medicine, Feinberg School of Medicine, Northwestern University, 676 St Clair St, Suite 1400, Chicago, Illinois 60611-2951, USA

Tel: +1-312-695-4729, Fax: +1-312-695-3999, E-mail: a-read@northwestern.edu

Financial support: This work was supported by R01 DK079902 (JEP) from the Public Health Service. Conflicts of interest: JEP worked as a consultant for Given Imaging, Sandhill Scientific, Crospon Inc. 
sus guidelines formed through an interdisciplinary panel have the potential to help standardize the diagnosis going forward. ${ }^{1}$ However, there has been minimal emphasis on the mechanical endpoints of the disease, which is surprising given that the primary determinant of symptom generation is focused on mechanical obstruction and a reduction in compliance due to inflammation, edema and fibrosis of the esophageal wall. This review will specifically address the biomechanical aspects of EoE and will focus on the pathogenesis and management of these perturbations of esophageal body function.

\section{Clinical Presentation of Eosinophilic Esophagitis}

While eosinophils had been previously described elsewhere in the gastrointestinal tract as eosinophilic gastroenteritis, the recognition of isolated eosinophilia within the esophagus associated with clinical symptoms of dysphagia has only recently emerged as a unique clinical entity. ${ }^{3}$ In the late 1970 s, case reports began to emerge of individuals with clinical symptoms of esophageal dysmotility and eosinophilic infiltrate on esophageal biopsy. ${ }^{4,5}$ In the 1980s, patients with gastroesophageal reflux disease (GERD) were noted to have eosinophilic esophageal infiltrates. ${ }^{6}$ In 1993, Attwood et al. described a new clinicopathologic syndrome of intraepithelial esophageal eosinophils in individuals with dysphagia, normal endoscopy and normal 24-hour esophageal $\mathrm{pH}$ monitoring. This syndrome was distinct from the eosinophilia reported in GERD and represented the disease that we now know as eosinophilic esophagitis. ${ }^{7}$ Since then, the incidence of EoE has been increasing. While the reasons for the increasing incidence are not certain, they may be related to increased awareness of the diagnosis and increased usage of esophagogastroduodenoscopy. ${ }^{8}$ A prospective 20 year study from Switzerland suggests that the incidence of EoE has been increasing, independent of esophagogastroduodenoscopy frequency within the region studied. ${ }^{9}$ With increasing incidence and limited disease specific mortality, the prevalence of $\mathrm{EoE}$ is also rising. One theory that may explain the rise in EoE prevalence focuses on the hygiene hypothesis, or the idea that improvements in sanitation and treatment of infectious disease have led to decreased exposure to microorganisms during childhood and subsequent increased incidence of allergic or autoimmune processes. ${ }^{10}$ The disease occurs more commonly in Caucasians, males, and patients with an atopic history (eg, asthma, eczema and allergic rhinitis). ${ }^{2,11}$

As a clinicopathologic diagnosis, EoE requires both clinical findings and specific pathological criteria for a diagnosis. Characteristic histopathologic changes in EoE include eosinophilic infiltration, basal zone hyperplasia and subepithelial fibrosis with increased collagen deposition. ${ }^{12}$ Clinically, common symptoms include food impaction, dysphagia, and chest or abdominal pain. Other conditions that can cause eosinophilic infiltration of the esophagus including Crohn's disease, parasitic infection, or GERD must be excluded for a diagnosis of EoE. ${ }^{3}$ It is particularly important to distinguish GERD from EoE as some cases of esophageal eosinophilia are entirely responsive to proton pump inhibitor (PPI) therapy; current guidelines exclude these patients from a diagnosis of EoE. ${ }^{13}$

Pathologically, for a diagnosis of EoE, at least 15 eosinophils per high powered field (hpf) are required. ${ }^{1}$ The finding of eosinophils in the esophagus is abnormal and their presence should alert the clinician to search for an etiology. ${ }^{14}$ However, the presence of eosinophils in the esophagus is necessary but not sufficient for the diagnosis of EoE, ie, there are other causes of esophageal eosinophilia. ${ }^{13}$ Current diagnostic criteria allow for the diagnosis of EoE in individuals with fewer than 15 eosinophils/hpf who have other abnormalities that are strongly associated with eosinophilic inflammation, such as eosinophilic microabscesses or extracellular eosinophil granules. ${ }^{1}$ The microscopic changes to the structure of the esophagus can manifest with gross structural changes that may suggest a diagnosis of EoE, including esophageal rings, furrows, whitish exudates or plaques and strictures. ${ }^{15,16}$ Esophageal rings can be fixed (also referred to as "esophageal trachealization") or transient (also described as "feline folds"). ${ }^{1,2}$ None of the endoscopic findings is pathognomonic for $\mathrm{EoE}$ and the absence of endoscopic features does not preclude the existence of EoE at biopsy. ${ }^{1} \mathrm{~A}$ recent meta-analysis has shown that although the majority of patients with EoE have an abnormal endoscopic appearance, the operating test characteristics of endoscopic changes alone are inadequate for use as sole diagnostic criteria, with a sensitivity of only $15 \%-48 \% .^{17}$

Although the diagnosis of EoE is heavily dependent on the above histologic changes, there is a disconnect between the degree of eosinophilia and symptom severity. Treatment trials have revealed a poor correlation between symptom response and eosinophil count and thus other factors must be assessed when managing patients with EoE. Given that the primary symptoms of dysphagia and food impaction are directly related to the degree of obstruction in the esophageal body, it would seem natural to shift some attention toward the assessment of the mechanical properties of the esophageal wall when one is assessing treatment 
outcomes. Although targeting the allergic and inflammatory process should still be a primary focus for therapeutic interventions, outcomes for treatment should also focus on improving the compliance of the esophageal wall and resolving the mechanical obstruction that drives symptom severity.

\section{Mechanisms Which May Alter Body Wall Mechanics}

In susceptible individuals, $\mathrm{EoE}$ is triggered by an immune response to antigens acquired via food (gastrointestinal tract) or inhalation ("aeroantigens," via the respiratory tract). ${ }^{13}$ Recognition of these antigens by the immune system leads to a series of cytokine-mediated inflammatory responses resulting in eosinophilic infiltration of the esophagus and alterations to the structure of the esophagus with basal zone hypertrophy. ${ }^{18}$ The results of these changes to the structure of the esophagus lead to functional changes that manifest as the clinical symptoms of EoE.

Eosinophils, myeloid-derived cells named for the prominent uptake of eosin within their intracellular granules, have been implicated in a variety of processes including defense against parasitic infections, asthma, atopic dermatitis and malignancy. ${ }^{19}$ Contained within the eosinophils are four cytotoxic granules that can cause cell death and tissue damage when released: major basic protein, eosinophilic cationic protein, eosinophilic peroxidase and eosinophilic derived neurotoxin. ${ }^{20}$ In EoE, an inflammatory response is triggered by Th2 cells in response to antigen, leading to production of IL-5 and IL-13. ${ }^{14,18}$ IL-5 activates eosinophils, which in turn release TGF- $\beta$, which stimulates fibroblasts and promotes fibrosis. ${ }^{14}$ IL-13 activates esophageal epithelial cells to upregulate eotaxin-3, a cell surface receptor that attracts eosinophils, and is the most highly induced gene in EoE. ${ }^{12,14}$ Mouse models of EoE have been created in which intratracheal IL-13 promotes development of esophageal eosinophilia. ${ }^{21}$

Epithelial mesenchymal transformation (EMT), or the series of changes in which epithelial cells lose their characteristic properties (polarity, surface markers and tight junctions etc) and transform into mesenchymal cells, has been implicated in the pathogenesis of fibrosis seen in EoE. ${ }^{22}$ EMT has been described in chronic disease processes in other organs including the kidney, liver, and lung and has been found in chronic asthma. The process of EMT in EoE appears to be driven by TGF- $\beta$, suggestive of an eosinophil-mediated process. ${ }^{22}$ Treatment of the underlying EoE disease process with immunosuppressants seems to reverse the progression of EMT, suggesting that some of the patho- genesis of EoE may be reversible. ${ }^{22}$

\section{Assessing Esophageal Body Wall Mechanics}

The biomechanical effects of the structural changes associated with EoE have been studied using impedance planimetry, barium esophagograms, manometry and endoscopic ultrasound. These tools have provided some insight into the pathogenesis of the disease and will likely provide the foundation for assessing objective outcomes of treatment.

\section{High-resolution Impedance Planimetry}

Kwiatek et $\mathrm{al}^{23}$ investigated the measurement of esophageal wall mechanics by measuring distensibility using the EndoFLIP ${ }^{\circledR}$ device. The EndoFLIP ${ }^{\circledR}$ system (or Endoscopic Functional Luminal Imaging Probe; Crospon Medical Devices, Galway, Ireland), is a device that utilizes impedance planimetry to determine distensibility within a hollow organ. The device contains a cylindrical bag that is placed in the esophagus and inflates with a conductive fluid, allowing simultaneous measurements of cross sectional areas and intrabag pressures which can be used to determine the distensibility of the esophagus (Fig. 1). ${ }^{23}$ Findings from this study were notable for a decrease in the extent of esophageal distensibility in patients with EoE versus control patients. ${ }^{23}$ Esophageal compliance was lower in the EoE patient group than the control group. Interestingly, distensibility was independent of the degree of esophageal eosinophilia, suggesting that changes to the structure of the esophagus may occur that are mediated by the eosinophils but independent of count, making it difficult to grade clinical severity by pathology alone. ${ }^{23}$ The structural changes underlying the changes in distensibility are likely related to the EMT promoted by the eosinophils and the Th2-mediated allergic response. Future studies should assess whether the changes in distensibility will improve with treatment of EoE.

\section{Barium Esophagogram}

A group at Mayo Clinic recently investigated esophageal properties in EoE using barium esophagograms to measure esophageal diameters. ${ }^{24}$ At baseline, about half of EoE patients had a decreased esophageal diameter. With steroid treatment, the maximal esophageal diameter increased in patients with $\mathrm{EoE}$ who had decreased esophageal diameters at baseline. Interestingly, half of the patients in this study did not have evidence of baseline changes in distensibility, suggesting that distensibility is not the 
A Control subject

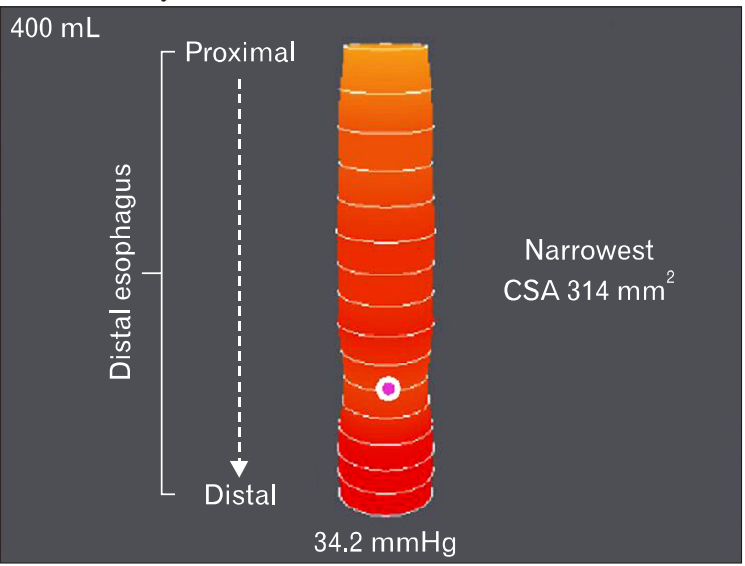

B EoE patient with narrowed distal esophagus

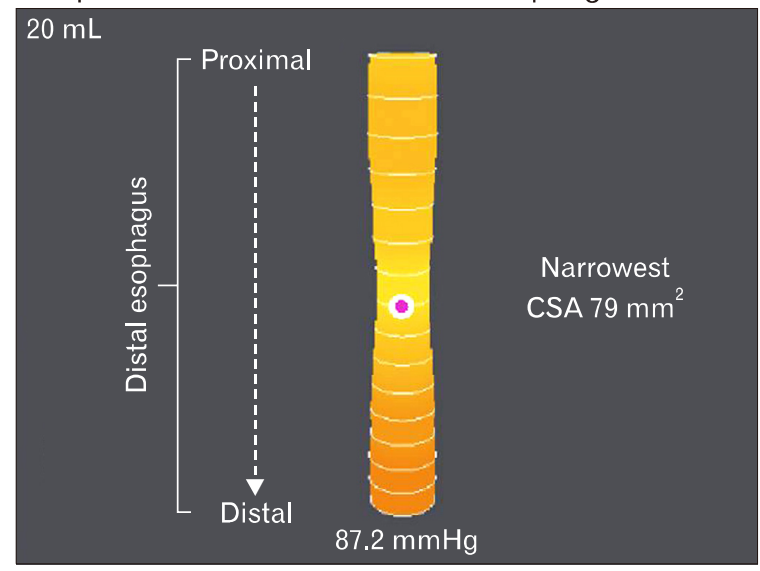

C EoE patient with a distal esophagus stricture

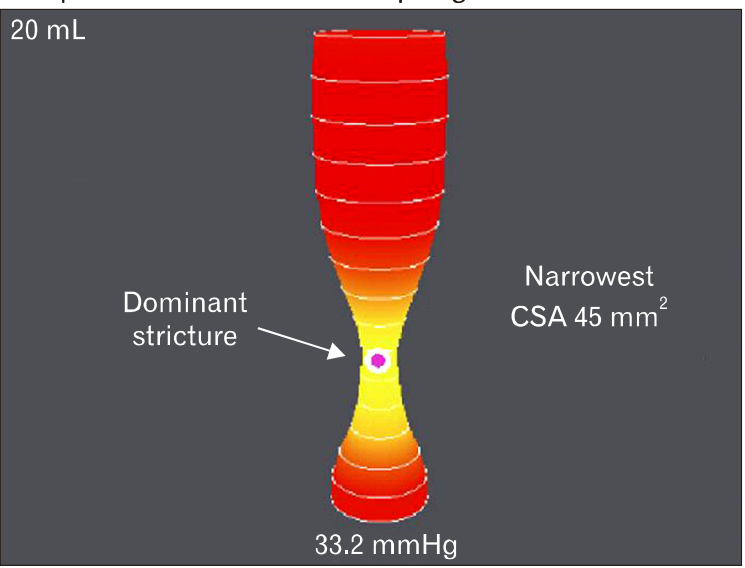

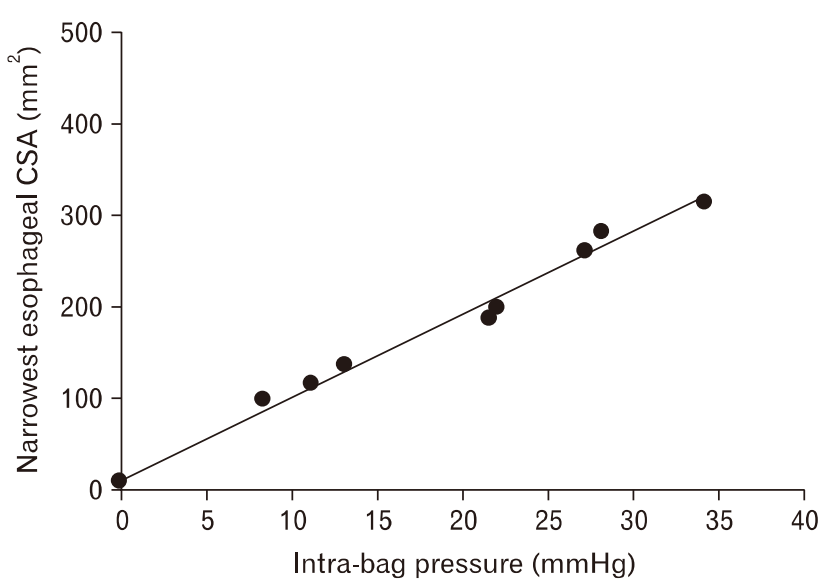
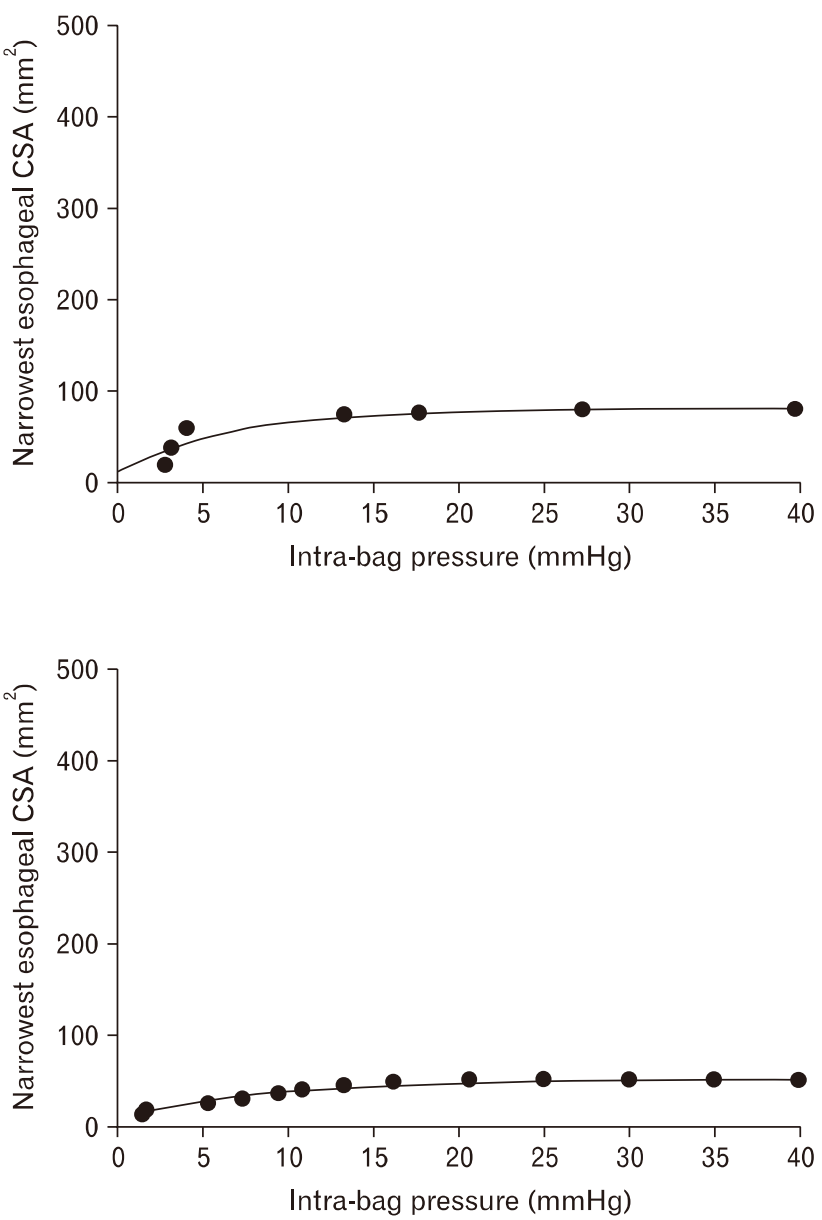

Figure 1. EndoFLIP ${ }^{\circledR}$ distensions. Sample EndoFLIP ${ }^{\circledR}$ distensions seen in a control subject (A), an EoE patient with a narrowed distal esophagus (B) and an EoE patient with a dominant distal esophageal stricture (C). Esophageal distension is displayed as a cylinder of varying diameter which corresponds to the cross-sectional areas (CSAs) measured by impendence planimetry technique and corresponding intra-bag pressure. The pink dot represents the narrowest CSA. Response to distention are plotted to right. Adapted from Kwiatek et al. ${ }^{23}$ 
sole explanation for dysphagia in EoE. ${ }^{24}$ However, this technique is somewhat limited by the fact that the esophagus is only evaluated using one plane and the true compliance or distensibility cannot be ascertained without a pressure measurement. Thus, it is unlikely that barium esophagograms will provide a useful measure of body wall function. However, the technique may be useful in the management of strictures and guiding dilation technique.

\section{Manometry}

If structural changes in the esophagus causing alteration in distensibility do not wholly explain the dysphagia symptoms of EoE, then perhaps there are changes in motor function as well. Multiple studies using esophageal manometry to study EoE have been published with no clear pathognomonic findings defined for EoE. In fact, studies have been variable in terms of the findings seen in eosinophilic esophagitis.

Lucendo et $\mathrm{al}^{25}$ found high-amplitude peristaltic waves in the distal two thirds of the esophagus. In a study using conventional manometry, Moawad et $\mathrm{al}^{26}$ found evidence of nutcracker esophagus in a subset of patients with EoE who had the highest mean eosinophil counts. However, this was a retrospective study looking at only manometry of patients with a diagnosis of EoE and thus did not have an adequate control group. ${ }^{26}$ It is also important to consider the possibility that some of the findings of severe dysphagia in individuals with EoE may be related to a concomitant motility disorder. Publication of a small case series may misattribute these symptoms to EoE when in fact there is a second process at work. To minimize bias and to control for background population motility disorders it is important to include a control group in these studies. In addition, an important clinical question is whether these manometric findings improve with treatment of EoE.

In a 2011 study, Roman et $\mathrm{al}^{27}$ examined high resolution manometry studies in patients with eosinophilic esophagitis, GERD patients and asymptomatic controls. This study demonstrated that increased bolus pressurization occurred more frequently in patients with EoE than in patients with GERD or control patients. There was an increased frequency of weak and failed peristalsis in EoE; however, this finding was also seen in GERD populations. Unique to EoE patients was early pan-esophageal pressurization, a finding which may be a manifestation of the reduced compliance in the esophagus seen by EndoFLIP ${ }^{\mathbb{R}}$ studies (Fig. 2). ${ }^{27}$ Using high-resolution manometry in EoE vs controls, Martín Martín et $\mathrm{al}^{28}$ similarly found a higher incidence of pan-esophageal pressurization in patients with EoE.
In 2011, Savarino et $\mathrm{al}^{29}$ reported a case of a 22-year-old man who presented with intermittent dysphagia, found to have normal endoscopy, manometric findings suggestive of achalasia and dense eosinophilic inflammatory infiltrate ( $>50$ eosinophils/hpf in peak density) on esophageal biopsy. The patient was treated with prednisolone and his symptoms resolved. Repeat manometry demonstrated normal peristalsis and subsequent biopsies demonstrated a resolution of the eosinophilic infiltrate. ${ }^{29}$ These findings suggest that some features of dysphagia seen in EoE are directly related to dysmotility. In addition, it raises the possibility that at least some patients diagnosed with achalasia may have an underlying diagnosis of $\mathrm{EoE}$ and thus may be steroid-responsive. As in this case, with a normal endoscopic appearance, it may be important to pursue random biopsies to look for $\mathrm{EoE}$ in cases of idiopathic achalasia as some of the diagnosed cases of achalasia may actually be secondary to EoE.

\section{Endoscopic Ultrasound}

Esophageal manometry primarily measures circular muscle activity. In EoE patients with normal circular muscle activity but symptoms of dysphagia something else must structurally be responsible for the subjective complaints of dysphagia and objective findings of food impaction. If the endoscopic appearance of the esophagus is normal and there are no strictures, something else intrinsic to the esophagus itself could be involved. In addition to circular muscle, esophageal smooth muscle also contains longi-

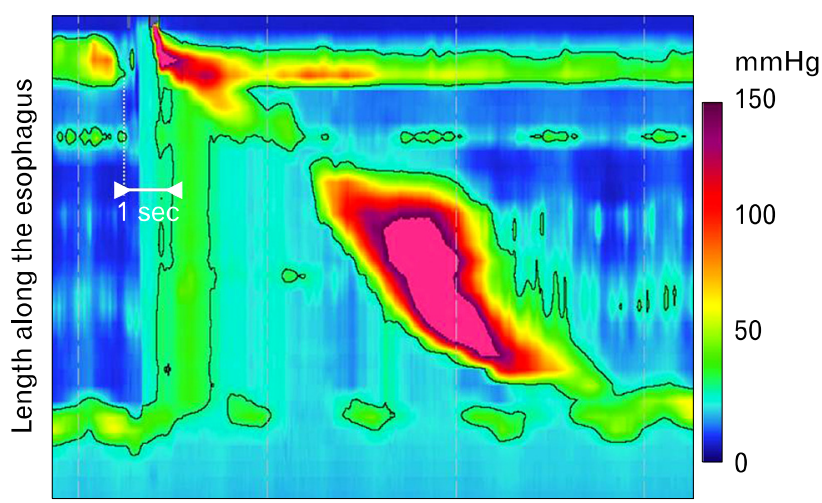

Figure 2. Early pan-esophageal pressurization pattern. An example of early pan-esophageal pressurization pattern seen with esophageal pressure topography, obtained by high-resolution manometry. The black line outlines an isobaric domain that includes all pressure signals above $30 \mathrm{mmHg}$. The early pan-esophageal pressurization is due to a stiff esophageal wall not expanding to accommodate the volume of liquid swallowed and this eventually resolves once emptying through a normal esophagogastric junction occurs. Adapted from Roman et al. ${ }^{27}$ 
tudinal muscle, which has been suggested as a putative culprit for the symptoms of dysphagia in EoE.

In a landmark 2009 study using simultaneous manometry and esophageal ultrasound, Korsapati et $\mathrm{al}^{30}$ demonstrated that patients with EoE had changes in the function of esophageal longitudinal muscles that may contribute to dysphagia. While traditional esophageal manometry primarily measures the function of circular muscles, Korsapati et $\mathrm{al}^{30}$ used endoscopic ultrasound to measure the function of the longitudinal muscles. The results of the study showed that circular muscle function (based on conventional manometry) was normal in the EoE patients but that longitudinal muscle function was severely diminished in the EoE patients, as demonstrated by decreased longitudinal muscle contraction amplitude and duration. EoE patients were noted to have dissociation between longitudinal and circular muscle contractions, whereas control patients had synchronous contractions between longitudinal and circular muscle contractions. ${ }^{30}$ Additionally, Korsapati et $\mathrm{al}^{30}$ used edrophonium, an acetylcholinesterase inhibitor, to determine the effects of increased acetylcholine levels at the neuromuscular junction. Control patients demonstrated an increase in circular muscle and longitudinal muscle contractions to edrophonium; EoE patients did not mount the same response. The authors hypothesized that the likely etiology of the dysfunction was the longitudinal muscle because the baseline circular muscle function was normal but a dissociation existed between the circular and longitudinal muscle function. ${ }^{30}$

Interesting questions were raised regarding the mechanisms causing longitudinal muscle dysfunction, the process by which longitudinal muscle dysfunction causes dysphagia, and the possibility that treatment might reverse the longitudinal muscle dysfunction. Of note, Korsapati et $\mathrm{al}^{30}$ showed in a subset analysis that three of the EoE patients who were undergoing treatment demonstrated lower amounts of longitudinal muscle dysfunction than those not on treatment; however, the sample size was too small to make statistically significant conclusions. Further studies with a larger sample size powered to detect treatment effects would be useful to further understand the biomechanics of EoE and response to treatment.

\section{Treatments Focused on Improving Body Wall Mechanics}

Clinically, treatments for EoE have included dietary avoidance of specific allergens and suppression of the immune response using either local steroids or systemic corticosteroid therapy. The mainstay of therapy for EoE can be remembered by the "3D's": diet, drugs and dilation. ${ }^{31}$ An understanding of the biomechanical changes of the esophagus is important to understand the ways these therapies work. Dietary therapy using an elimination diet has been shown to reduce symptoms and reduce eosinophilic infiltrate of the esophagus, by reducing the antigenic stimulus to the Th2-mediated response. ${ }^{32}$ Drug therapies attempt to interrupt the inflammatory cascade using systemic glucocorticoids, localized steroids (swallowed or inhaled) and biologic agents. ${ }^{33}$ Mepolizumab, a monoclonal antibody against IL-5, has been shown to reduce esophageal eosinophilia in pediatric patients with EoE. ${ }^{34}$ In a mouse model of EoE, monoclonal antibodies to IL-13 were shown to interfere with IL-13 mediated promotion of EoE pathogenesis, but the drug is not yet in clinical trials in humans. ${ }^{35}$ Although these treatments will reduce the inflammatory process, there is little evidence to support that they directly treat the obstruction or strictures associated with EoE.

The only therapy that directly targets treating the underlying obstruction is dilation therapy. Mechanical esophageal dilation therapy has been used successfully in patients with strictures in EoE, although medical therapy is generally preferred as first line therapy due to concerns of increased friability of the mucosa in EoE and concerns about an increased risk of perforation. ${ }^{33} \mathrm{~A}$ systematic review has shown that the incidence of true perforation is rare. ${ }^{36}$ While post-procedural pain is common in the short run, the majority of patients with EoE who undergo dilation experience improvement in their symptoms of dysphagia. ${ }^{36}$ The most significant limitation of dilation therapy in $\mathrm{EoE}$ is that it does not appear to affect the underlying immunopathogenesis of the disease. ${ }^{31}$ As a clinicopathologic syndrome, it is important to carefully consider the endpoints used in clinical trials and differentiate between clinical improvement in symptoms and histopathologic changes of decreased eosinophilia on biopsy. A combined treatment approach that addresses factors that trigger the disease (diet), interrupts pathogenesis (drugs) and improves structural complications (dilation) may be needed to fully treat the disease and prevent worsening fibrosis and mechanical abnormalities.

\section{Conclusion}

EoE is an important emerging disease. The most important symptoms of EoE are food impaction and symptoms of dysphagia. Inflammatory changes that occur in EoE lead to esophageal fibrosis and an alteration of the biomechanical properties of 
the esophagus that can predispose to dysphagia and impactions. Studying mechanics should be a major focus of research into $\mathrm{EoE}$ as the predominant symptoms of EoE are directly related to changes that occur secondary to remodeling of the esophagus, including compliance and possibly motor function.

In this paper, we have reviewed some of the literature discussing esophageal biomechanics in EoE. Studies have explored the properties of the esophagus using a variety of techniques including manometry, endoscopic ultrasound, barium esophagograms and impedance planimetry. These studies have helped to elucidate the mechanisms of esophageal dysfunction seen in EoE from the cellular level to the endoscopic appearance of the esophagus. Diagnosis of EoE is challenging because it requires a combination of clinical and pathologic features and there is no one pathognomonic diagnostic test. An understanding of the cellular, molecular and genetic basis for the immunopathogenesis of $\mathrm{EoE}$ is important to help develop treatments that can interrupt the process by targeting key components of the inflammatory cascade. Measuring the biomechanics in EoE may be important to help determine objective surrogate endpoints for clinical trials of therapies as reliance upon symptom scoring alone or eosinophil count for response to treatment may ignore the most important underlying mechanism for symptoms. We believe EoE should be conceptualized similar to Crohn's disease where the presenting phenotypes may be dominated by either fibrostenotic changes or inflammatory changes. These phenotypes would likely require different approaches and an assessment of mechanics could help tailor therapy.

\section{Acknowledgements}

AJR and JEP performed the research and wrote the paper.

\section{References}

1. Liacouras CA, Furuta GT, Hirano I, et al. Eosinophilic esophagitis: updated consensus recommendations for children and adults. J Allergy Clin Immunol 2011;128:3-20. e6.

2. Dellon ES. Eosinophilic esophagitis: diagnostic tests and criteria. Curr Opin Gastroenterol 2012;28:382-388.

3. Rothenberg ME. Eosinophilic gastrointestinal disorders (EGID). J Allergy Clin Immunol 2004;113:11-28.

4. Dobbins JW, Sheahan DG, Behar J. Eosinophilic gastroenteritis with esophageal involvement. Gastroenterology 1977;72:1312-1316.

5. Landres RT, Kuster GG, Strum WB. Eosinophilic esophagitis in a patient with vigorous achalasia. Gastroenterology 1978;74:12981301.
6. Winter HS, Madara JL, Stafford RJ, Grand RJ, Quinlan JE, Goldman H. Intraepithelial eosinophils: a new diagnostic criterion for reflux esophagitis. Gastroenterology 1982;83:818-823.

7. Attwood SE, Smyrk TC, Demeester TR, Jones JB. Esophageal eosinophilia with dysphagia. A distinct clinicopathologic syndrome. Dig Dis Sci 1993;38:109-116.

8. Prasad GA, Alexander JA, Schleck CD, et al. Epidemiology of eosinophilic esophagitis over three decades in Olmsted County, Minnesota. Clin Gastroenterol Hepatol 2009;7:1055-1061.

9. Hruz P, Straumann A, Bussmann C, et al. Escalating incidence of eosinophilic esophagitis: a 20-year prospective, population-based study in Olten County, Switzerland. J Allergy Clin Immunol 2011; 128:1349-1350. e5.

10. Lucendo AJ, Lucendo B. An update on the immunopathogenesis of eosinophilic esophagitis. Expert Rev Gastroenterol Hepatol 2010;4: 141-148.

11. Remedios M, Jones D, Kerlin P. Eosinophilic oesophagitis: epidemiology, pathogenesis and management. Drugs 2011;71:527-540.

12. Tourangeau LM, Aceves SS. Esophageal remodeling in eosinophilic esophagitis. In: Liacouras CA, Markowitz JE, eds. Eosinophilic esophagitis. New York: Humana Press 2012:83-96.

13. Abonia JP, Rothenberg ME. Eosinophilic esophagitis: rapidly advancing insights. Annu Rev Med 2012;63:421-434.

14. Rothenberg ME. Biology and treatment of eosinophilic esophagitis. Gastroenterology 2009;137:1238-1249.

15. Sherrill JD, Rothenberg ME. Genetic dissection of eosinophilic esophagitis provides insight into disease pathogenesis and treatment strategies. J Allergy Clin Immunol 2011;128:23-32.

16. Veerappan GR, Perry JL, Duncan TJ, et al. Prevalence of eosinophilic esophagitis in an adult population undergoing upper endoscopy: a prospective study. Clin Gastroenterol Hepatol 2009;7:420426, 426. e1-e2.

17. Kim HP, Vance RB, Shaheen NJ, Dellon ES. The prevalence and diagnostic utility of endoscopic features of eosinophilic esophagitis: a meta-analysis. Clin Gastroenterol Hepatol 2012;10:988-996. e5.

18. Aceves SS, Ackerman SJ. Relationships between eosinophilic inflammation, tissue remodeling, and fibrosis in eosinophilic esophagitis. Immunol Allergy Clin North Am 2009;29:197-211, xiii-xiv.

19. Blanchard C, Rothenberg ME. Biology of the eosinophil. Adv Immunol 2009;101:81-121.

20. Rothenberg ME, Hogan SP. The eosinophil. Annu Rev Immunol 2006;24:147-174.

21. Mulder DJ, Justinich CJ. Understanding eosinophilic esophagitis: the cellular and molecular mechanisms of an emerging disease. Mucosal Immunol 2011;4:139-147.

22. Kagalwalla AF, Akhtar N, Woodruff SA, et al. Eosinophilic esophagitis: epithelial mesenchymal transition contributes to esophageal remodeling and reverses with treatment. J Allergy Clin Immunol 2012;129:1387-1396. e7.

23. Kwiatek MA, Hirano I, Kahrilas PJ, Rothe J, Luger D, Pandolfino JE. Mechanical properties of the esophagus in eosinophilic esophagitis. Gastroenterology 2011;140:82-90.

24. Lee J, Huprich J, Kujath C, et al. Esophageal diameter is decreased in some patients with eosinophilic esophagitis and might increase with topical corticosteroid therapy. Clin Gastroenterol Hepatol 2012;10: 481-486. 
25. Lucendo AJ, Pascual-Turrión JM, Navarro M, et al. Endoscopic, bioptic, and manometric findings in eosinophilic esophagitis before and after steroid therapy: a case series. Endoscopy 2007;39:765-771.

26. Moawad FJ, Maydonovitch CL, Veerappan GR, Bassett JT, Lake JM, Wong RK. Esophageal motor disorders in adults with eosinophilic esophagitis. Dig Dis Sci 2011;56:1427-1431.

27. Roman S, Hirano I, Kwiatek MA, et al. Manometric features of eosinophilic esophagitis in esophageal pressure topography. Neurogastroenterol Motil 2011;23:208-214. e111.

28. Martín Martín L, Santander C, Lopez Martín MC, et al. Esophageal motor abnormalities in eosinophilic esophagitis identified by high-resolution manometry. J Gastroenterol Hepatol 2011;26: $1447-1450$

29. Savarino E, Gemignani L, Zentilin P, et al. Achalasia with dense eosinophilic infiltrate responds to steroid therapy. Clin Gastroenterol Hepatol 2011;9:1104-1106.

30. Korsapati H, Babaei A, Bhargava V, Dohil R, Quin A, Mittal RK. Dysfunction of the longitudinal muscles of the oesophagus in eosinophilic oesophagitis. Gut 2009;58:1056-1062.

31. Straumann A, Aceves SS, Blanchard C, et al. Pediatric and adult eo- sinophilic esophagitis: similarities and differences. Allergy 2012;67: 477-490.

32. Gonsalves N, Yang GY, Doerfler B, Ritz S, Ditto AM, Hirano I. Elimination diet effectively treats eosinophilic esophagitis in adults; food reintroduction identifies causative factors. Gastroenterology 2012;142:1451-1459. e1.

33. Almansa C, Devault KR, Achem SR. A comprehensive review of eosinophilic esophagitis in adults. J Clin Gastroenterol 2011;45:658664.

34. Assa'ad AH, Gupta SK, Collins MH, et al. An antibody against IL-5 reduces numbers of esophageal intraepithelial eosinophils in children with eosinophilic esophagitis. Gastroenterology 2011;141: 1593-1604.

35. Blanchard C, Mishra A, Saito-Akei H, Monk P, Anderson I, Rothenberg ME. Inhibition of human interleukin-13-induced respiratory and oesophageal inflammation by anti-human-interleukin-13 antibody (CAT-354). Clin Exp Allergy 2005;35:1096-1103.

36. Bohm ME, Richter JE. Review article: oesophageal dilation in adults with eosinophilic oesophagitis. Aliment Pharmacol Ther 2011;33: 748-757. 